\title{
Effect of Interfacial Structure on Gold-assisted Growth of Oxides
}

\author{
Xin $\mathrm{Li}^{1}$, Wei Zhou ${ }^{1}$, Fang Liu ${ }^{1}$ and Guo-zhen Zhu ${ }^{1}$
}

1. State Key Laboratory of Metal Matrix Composites, School of Materials Science and Engineering, Shanghai Jiao Tong University, 800 Dongchuan Rd., Shanghai, 200240, P. R. China.

Heterogeneous catalysts, e.g. $\mathrm{Au} / \mathrm{Fe}_{2} \mathrm{O}_{3}, \mathrm{Au} / \mathrm{TiO}_{2}$, and $\mathrm{Au} / \mathrm{MgAl}_{2} \mathrm{O}_{4}$, have profound significance for many energy-related reactions such as low-temperature $\mathrm{CO}$ oxidation and selective oxidation of alcohols. [1-2] In spite of their technological and scientific importance, the underlying catalytic mechanism remains largely unclear in literature. Many plausible hypotheses are related to the Au/oxide interface, which either plays a role in controlling the morphology of Au nanoparticles or has a direct contribution to their catalytic behaviors. [3-5] Nevertheless, it is crucial to fully clarify the interfacial structure, including the atomic arrangement and chemical bonding nature. In order to facilitate structural characterization, we synthesized model systems with Au nanoparticles on a single-crystal oxide support by the presence of an Au overlayer and the application of heat.

A new phenomenon, we have discovered recently in those systems, involves the dewetted gold nanoparticle acting as a seed causing crystalline substrates to spontaneously grow underneath the gold nanoparticle.[5] Fig.1 shows intricate nano-structures including dewetted gold nanoparticles supporting by the regrowth oxide bases. As shown in the HAADF (Z-contrast) images, gold nanoparticles, with well-defined facets on crystalline bases, share the same contrast with the substrate. The composition and the crystallographic orientation of the bases are further confirmed by the electron energy-loss spectroscopy and diffraction techniques, respectively. The formation of such intricate nanostructures depends on a few parameters such as the thickness of the Au overlayer and the heating treatment.

Limited to the $\mathrm{Au}-\mathrm{TiO}_{2}$ and $\mathrm{Au}-\mathrm{MgAl}_{2} \mathrm{O}_{4}$ systems we have studied so far, the intricate nano-structures include: a). gold nanoparticles, normally close to the Wulff equilibrium shape, have a few preferential crystallographic orientations with respect to the single-crystal substrates; b). regrowth oxide bases, with a well-defined shape, are epitaxially aligned with the single-crystal substrates; and c). interfacial monolayers, which have completely different atomic arrangement, extends both the gold and oxide lattices. The formation of such interfacial monolayers always accompanies with the regrowth of the substrates. Those unique interfacial structures may be responsible for the epitaxial growth of the substrate under self-organized gold nanoparticles.

The interfacial monolayers referred as rearranged $\mathrm{Au}$ atoms between the gold and oxide lattices. We detected an interfacial bilayer between the gold nanoparticles and spinel bases, labeled by the red and blue arrows in Fig.2. On top of monolayer A with Au atoms extending the spinel lattice, the monolayer $\mathrm{B}$ exhibits an oscillating contrast in the HAADF image when viewed from $[110]_{\mathrm{MgAl} 2 \mathrm{O} 4}$. In the case of $\mathrm{Au}-\mathrm{TiO}_{2}$ with much large lattice mismatch, different interfacial structures have been observed with different crystallographic orientations between the gold and rutile lattice. Fig.3 shows the interfacial monolayers varies from single monolayer to a few monolayers viewed from $[001]_{\mathrm{TiO} 2}$.

The detailed atomic arrangement and chemical bonding nature of these interfacial monolayers are currently being investigated by a scanning TEM. The clarification of such structures provides deep insights in the understanding of the abnormal growth of the substrate. We believe that such phenomenon 
may shed the light on understanding the catalytic behavior of gold-oxide heterogeneous systems.

References:

[1] M. Haruta, S. Tsubota et. al., J. Catal. 144 (1993) p.175.

[2] C.H. Christensen, B. Jørgensen et al., Angew. Chem. Int. Ed. 45 (2006) p.4648.

[3] N. Lopez and J. K. Nørskov, J. Am. Chem. Soc. 124 (2002) p.11262.

[4] A.A. Herzing, C.J. Kiely, et. al., Science, 321 (2008) p.1331.

[5] J.A. Rodriguez, S. Ma, et. al., Science, 318 (2007) p.1757.

[6] G.-z. Zhu, T. Majdi, et. al., Appl. Phys. Lett. 105(2014) p.231607

[7] The authors acknowledge funding from the National Natural Science Foundation of China. Part of the microscopy work was carried out at Canadian Centre for Electron Microscopy (CCEM).

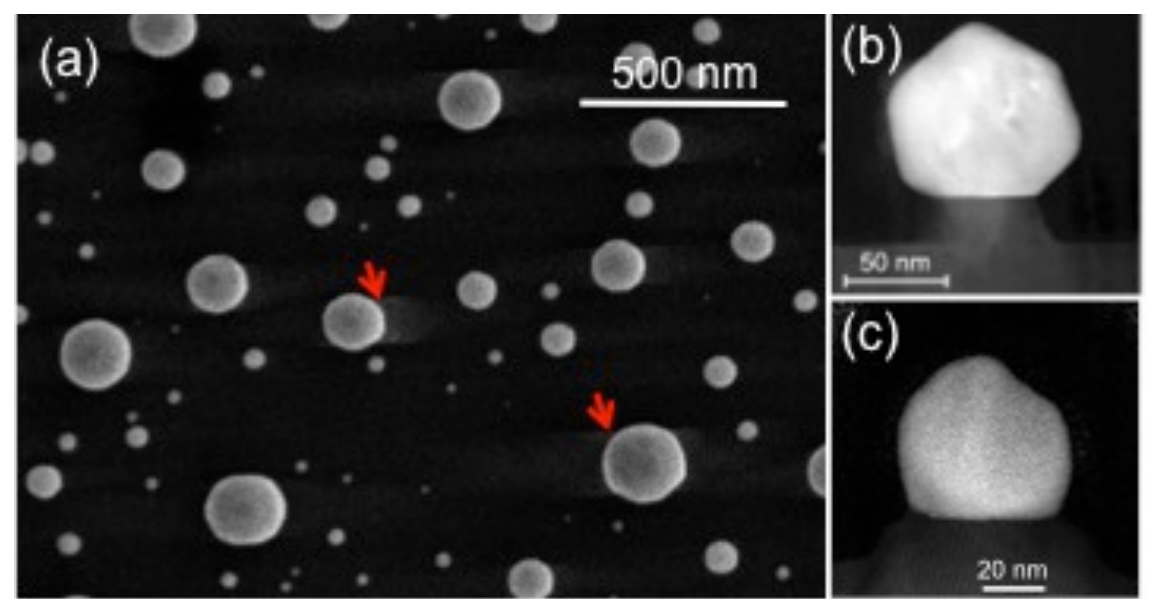

Figure 1. Intricately shaped gold-oxide nanostructures. (a) The $\mathrm{SEM}$ image of $\mathrm{Au}-\mathrm{TiO}_{2}$ nanostructure. The red arrows show the crystalline oxide bases supporting Au nanoparticles. (b) and (c) are the HAADF (Z-contrast) images presenting the cross-sectional view of the intricate nanostructure in Au$\mathrm{MgAl}_{2} \mathrm{O}_{4}$ and $\mathrm{Au}-\mathrm{TiO}_{2}$ systems, respectively.

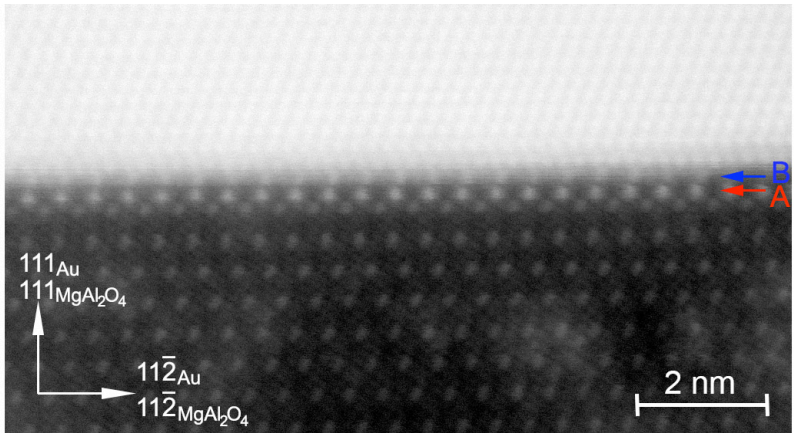

Figure 2. The $\mathrm{Au}-\mathrm{MgAl}_{2} \mathrm{O}_{4}$ interfacial bilayer.

Figure 3. The $\mathrm{Au}-\mathrm{TiO}_{2}$ interfacial bilayer. (a) and (b) show interfacial monolayers with different gold nanoparticles, respectively.
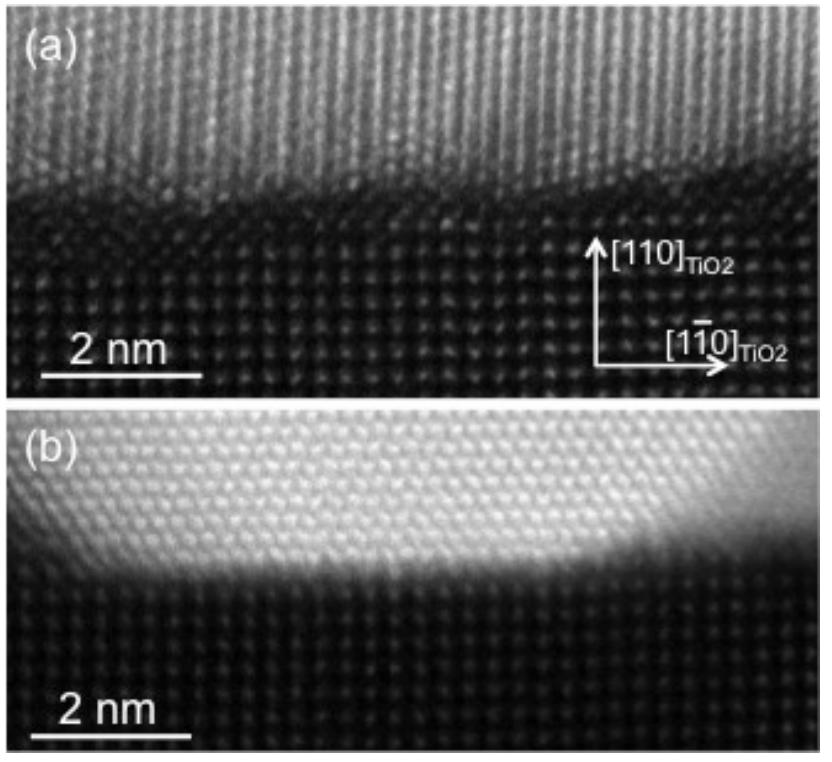